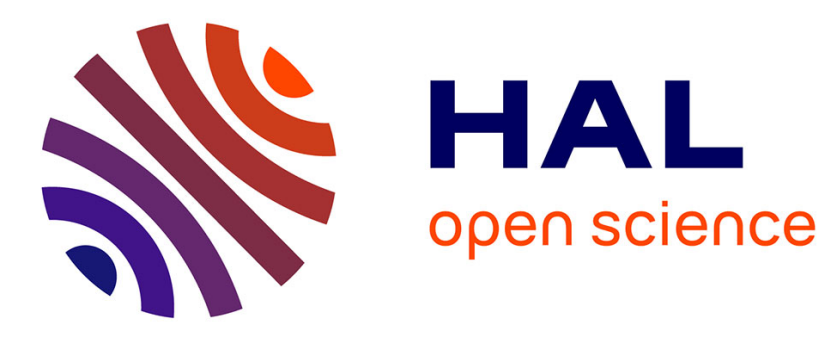

\title{
Pmcmtp's Implementation (in nesC/TinyOS2.x) and Testbed for Its Operation Validation
}

Jamila Ben Sliman, Ye-Qiong Song, Anis Koubaa, Mounir Frikha, Hugo Cruz Sanchez

\section{- To cite this version:}

Jamila Ben Sliman, Ye-Qiong Song, Anis Koubaa, Mounir Frikha, Hugo Cruz Sanchez. Pmcmtp's Implementation (in nesC/TinyOS2.x) and Testbed for Its Operation Validation. Wireless Sensor Networks - WSN 2011 (4th IFIP International Conference on New Technologies, Mobility and Security - NTMS 2011), Feb 2011, Paris, France. pp.1-6, 10.1109/NTMS.2011.5720636 . inria-00584105

\section{HAL Id: inria-00584105 https://hal.inria.fr/inria-00584105}

Submitted on 7 Apr 2011

HAL is a multi-disciplinary open access archive for the deposit and dissemination of scientific research documents, whether they are published or not. The documents may come from teaching and research institutions in France or abroad, or from public or private research centers.
L'archive ouverte pluridisciplinaire HAL, est destinée au dépôt et à la diffusion de documents scientifiques de niveau recherche, publiés ou non, émanant des établissements d'enseignement et de recherche français ou étrangers, des laboratoires publics ou privés. 


\title{
PMCMTP's implementation (in nesC/TinyOS2.x) and Testbed for its operation validation
}

\author{
Jamila Ben Slimane* ${ }^{*}$, Ye-Qiong Song ${ }^{\ddagger}$, Anis Koubâa ${ }^{\S}$, Mounir Frikha* and Hugo Cruz-Sanchez ${ }^{\ddagger}$ \\ * Sup'Com-MEDIATRON, University of $7^{\text {th }}$ November at Carthage, Tunisia \\ $\ddagger$ LORIA and INPL, Campus Scientifique, BP 23954506 Vandoeuvre-les-Nancy, France \\ ${ }^{\S}$ Cooperative Intelligent Networking Systems Research Group, Al-Imam Muhammad ibn Saud University, \\ Computer Science Dept., 11681 Riyadh, Saudi Arabia \\ Email: jamilabs07@yahoo.fr, Song@loria.fr, akoubaa@dei.isep.ipp.pt, m.frikha@ supcom.rnu.tn, Hugo.Cruzsanchez@loria.fr
}

\begin{abstract}
PMCMTP is a Prioritized Multi-Channel MultiTime slot MAC protocol that the authors have proposed for allowing to simultaneous use of several frequency channels. This protocol is designed for UWB of IEEE802.15.4a but it can also be used over IEEE802.15.4. In this paper, we design and implement a testbed of this protocol to demonstrate its practical implementability. Due to the unavailability of UWB transceiver, the testbed has been performed using classic $2.4 \mathrm{GHz}$ WSN transceivers. To reduce the complexity of resource sharing, the global network is composed of a set of Personal Area Networks (PANs) or cells. So, the PMCMTPs experiments are performed for a single PAN and two PANs.
\end{abstract}

\section{Keywords-WSN; PMCMTP; Testbed.}

\section{INTRODUCTION}

Wireless sensor networks may consist of a collection of spatially distributed autonomous sensor nodes characterized by limited memory, processing capability and battery power supply. Such networks represent an emerging technology with wide range of potential applications such as medical systems, environment monitoring, military applications, etc. An important fact that can not be neglected in WSNs is the need of quality-of-service (QoS) support. According to novel application requirements, QoS constraints become more and more critical in terms of end-to-end delay and data throughput. Also, due to energetic constraints at node level, energy saving remains the most challenging issue. Both IEEE 802.15.4 and its recent amendment IEEE 802.15.4a [1] standards allow dynamic channel allocation and use of multiple channels available at their physical layers but their MAC protocols are designed only for single channel. Also, sensor's transceivers such as CC2420 used by current WSN hardware (MICAZ, TelosB, and CMU FireFly), provide multiple channels, and as shown in [2] and [3], channel switch latency of CC2420 transceiver is short (just about $200 \mu s$ ). So this gives the possibility to simultaneous use of multi-channels, greatly increasing data throughput.

The idea of multi-channel MAC protocols is not new in the wireless network research community. Recently some MAC layer multi-channel protocols have been proposed to improve

This paper has been accepted for publication in the proceedings of WSN Workshop that had been held in conjunction with NTMS 2011. network performance in WSNs [4]-[8]. But, the majority of the proposed protocols were tested and evaluated by simulations, using personal or academic or commercial network simulator, and only protocols proposed in [7] and [8] were validated and evaluated by testbed deployed in a realistic setting.

In this paper, we present the implementation of a Prioritized Multi-Channel Multi-Time slot media access control Protocol (PMCMTP) [9] for WSNs. We, experimentally, validate the PMCMTP by using a realistic testbed setting.

\section{RELATED WORK}

The WSNs research is currently gaining an exponentially increasing interest from both academia and industry. New application scenarios, MAC, routing and physical layer optimizing protocols and algorithms are being developed by the research community. The ultimate test for the validity of these research activities is to implement the various scenarios, algorithms and protocols on a testbed and deploy it in a realistic setting.

Several testbeds have been designed for the experimentation of WSNs [7], [8], [10]-[12]. We can distinguish two class of testbeds: the first for general WSNs tests and the second for specific WSNs tests. MoteLab [10] is a web-based WSN testbed consisting of a set of MicaZ motes connected to a central server. Its goal is to allow users to evaluate WSN applications without manually re-programming and redeploying the nodes into the physical environment. Emulab [11] is a remotely accessible mobile and wireless sensor testbed. It can be used to study network topologies, mobility effects on protocols, test algorithms, and mobile applications. So, motes are deployed in mobile robots and users can create experiments through a web interface and schedule events to control the robots movement. The goal of such category of WSNs testbeds is to allow a large community of users (with different research goals) to share access to the testbed platform. So, resource are shared between users and it can not be available any time also testing environment is not manageable which. Such testebeds are useful for general WSNs tests by not for a specific WSNs tests. For a specific tests, in [12], the authors proposed their own testbed for the performance evaluation of link quality estimators. In [7] and [8], the proposed WSNs tesbeds targeted 
multi-channel WSN MAC protocols, to test respectively a multi-radio multi-channel MAC protocol and a mono-radio multi-channel MAC protocol. In this paper, we propose a WSN testbed to test the validity of our mono-radio multi-time slots multi-channel MAC protocol (PMCMTP).

Our paper makes the following main contributions:

- First, we implemented the PMCMTP in the nesC [13] programming language for MicaZ [14] motes with TinyOS2.x. [15]

- Second, we deployed a WSN testbed to ensure the experimental validation of the proposed protocol,

The rest of the paper is organized as follows: In Section 3, we present the system model. Section 4 gives an overview of the PMCMTP protocol. Section 5 gives a brief overview of the implementation of the PMCMTP on the MicaZ motes. In Section 6, we validate and demonstrate the effective operation of the PMCMTP and evaluate its performance by analyzing and commenting some experimental results.

\section{SYSTEM MODEL}

We have proposed in [16] the WHSN (Wireless Hospital Sensor Network) for an application in hospital (medical monitoring of patients and management of doctors). The WHSN is a three-tiered network, using UWB sensors in the first and second network levels and WiFi technology for the third tier (See Figure 1). The different network tiers are as follows:

1) First tier or the BSN (Body Sensor Network): a star network composed of one coordinator and a set of biosensors that ensure physiological measurements and the medical monitoring of patient.

2) Second tier or the PAN (Personal Area Network): a hexagonal cell of sensors organized in a full mesh topology including one PAN's coordinator, several mobile BSNs coordinators (one coordinator per BSN) and several routers.

3) Third tier or the Cellular Mesh WiFi Network: based on UWB/Wifi technologies, is chosen to the third level to have finally a three-tier hierarchical cellular network.

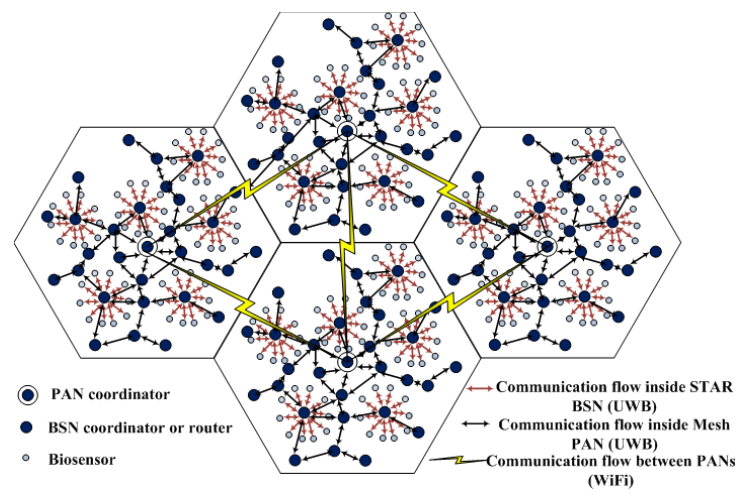

Fig. 1. Network architecture

The detailed description of the global network architecture is out of the scope of this paper, so for more details, one can refer to [16]. We focus in this paper to the second tier or the PAN. So, we consider a set of PANs organized on hexagonal cells to facilitate the spectrum resource allocation. Each PAN is composed by one coordinator and a set of router and/or source nodes. A source node can act as a BSN coordinator or a simple node. To provide time guarantee to deliver data frames, beacon-enabled mode is used. The format of the superframe is defined by the PAN Coordinator. The superframe, corresponding to the Beacon Interval $(B I)$, is defined by the time between two consecutive beacons, and includes an active period and, optionally, a following inactive period. The active period, corresponding to the Superframe Duration $(S D)$, is divided into 16 equally sized time slots, during which data transmission is allowed. For a global network of $N_{c}$ PANs, each PAN coordinator is characterized by its superframe duration $\left\{P A N_{i}=\left(S D_{i}, B I_{i}\right)\right\}_{1 \leq i \leq N_{c}}$ as shown in Figure 2. We define $\overline{B I_{m a j}}$ and $\overline{S D_{\min }}$ as respectively the major cycle (the least common multiple of all PANs $B I$ ) and the elementary active cycle (the least common denominator of all PANs $S D$ ).

\section{PMCMTP FOR IR UWB SENSOR NETWORKS}

In [17], we have proposed a channel allocation scheme to statically assign one control channel to each PAN to support the control traffic and dynamically share the set of available data channels between active PANs to support data traffic. The detailed description of the proposed channels (Control and data channels) sharing between PANs is out of the scope of this paper, so for more details, one can refer to [17]. So, let us assume that each PAN permanently dispose a control channel to ensure control traffic and a variable set of data channels to ensure data traffic. In this section, we focus on the detail of the PMCMTP [4] for logical channels and time slots allocation inside each active PAN. Similar to [18], a key concept in PMCMTP is the elementary active cycle, which is composed by two consecutive active periods, the first for synchronization and collection of resource requests, and the second for the Request Scheduling Algorithm (RSA) process, the reception of the second beacon and the allowable data communications (See Figure 3).

Inside each PAN, according to the PMCMTP, the PAN coordinator collects all the resource allocation requests of its sources nodes. Then, according to the available spectrum resource, it tries to allocate available time slots per available channel of frequencies in response to the collected requests. Finally, concerned sensors can start their data communication. The principle of PMCMTP is based on the three following phases:

1) Synchronization and Request Transmission: Transmission requests phase must precede each PAN's data communication active period. This phase is divided into two substeps :

(i) the first step consists in PAN synchronization: by listening to the beacon frame, PAN members adjust their wake-up clocks,

(ii) the second step consists in collecting all transmission 
requests from PAN's members. This step represents a set of equal short time slots, during which, the PAN coordinator is listening to the requests of PAN's member. So, just following the reception of the first beacon frame, each PAN's member waits for its own time slot in order to send its request packets. During this phase, the allocated control channel is used.

2) Channels/time slots allocation: According to the $R S A$ algorithm, after reception of all transmission requests, the PAN coordinator tries to schedule them according to their assigned priority. Once the list of requests is scheduled, the PAN coordinator tries to launch the phase of time slots and channels allocation. For each request, it tries to find the earliest available time slots per channel to assign it to the suitable request. At the end of the process of time slots and channels allocation, the PAN coordinator registers a trace of the requests not served in its queue in order to analyze them during the next cycle. Then, it inserts into the next beacon frame the necessary information (Request identifier, index of allocated channel, index of the first allocated time slot, number of allocated time slots, address of the request's sender and of flow destination) of the served requests.

3) Data communication: After listening to the second beacon frame, PAN's members can have a feedback of their transmission requests. Each concerned sensor switches to the suitable channel at the suitable time slot and begins sending or receiving data frames during the reserved duration.

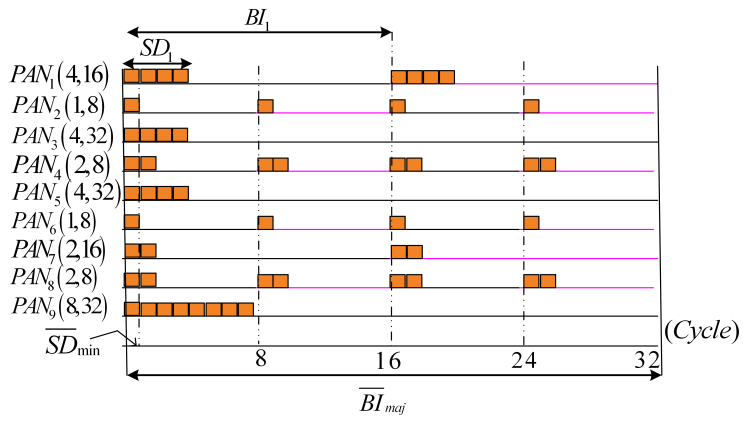

Fig. 2. Network configuration

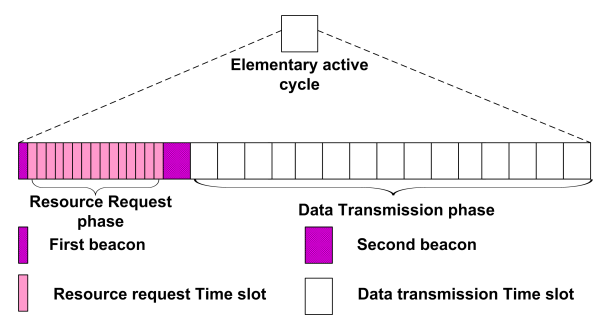

Fig. 3. An elementary active cycle

Figure 4 gives an example of a network using PMCMTP whose detail will be explained in the next section.

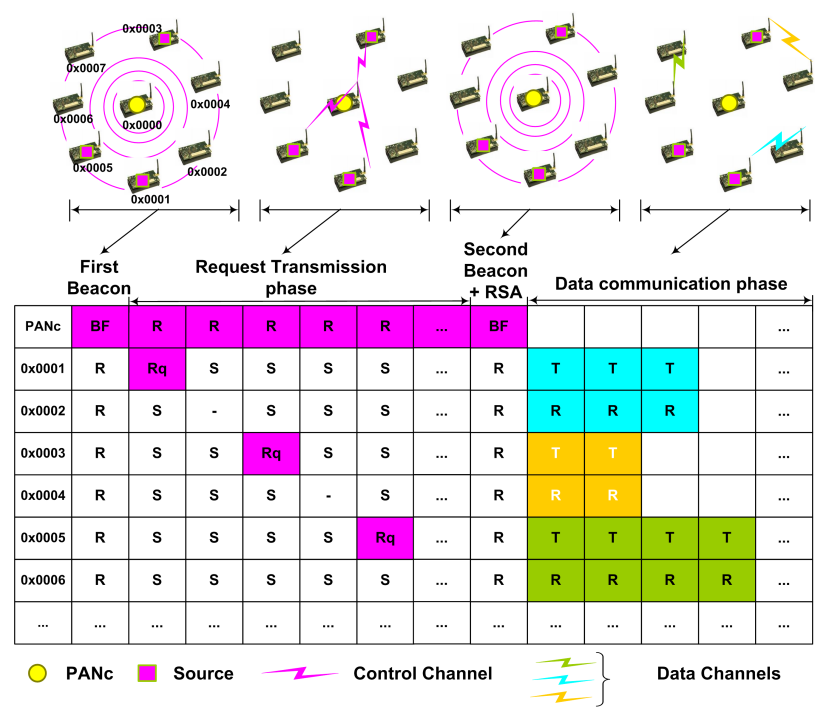

Fig. 4. PMCMTP process during an elementary active cycle

\section{IMPLEMENTATION OF PMCMTP IN NESC/TINYOS2.X}

Communication protocols and techniques are often tested and evaluated using simulation techniques. However, the use of realistic testbed setting for verification and evaluation in particular for WSNs becomes a necessity. In this part, we focus on the PMCMTP implementation in the nesC programming language for MICAz motes with TinyOS-2.x for the use in a real-world testbed setting to justify its operation validity in a real environment. The hardware used for the implementation of the PMCMTP protocol is the Crossbow MICAz motes, operating in the $2.4 \mathrm{GHz}$ ISM band. The MIB510 [19] programming board was used to program the motes. The PMCMTP's implementation is composed of two components Coordiator $C$ and DeviceC respectively implemented at the coordinator and device motes and an interface PMCMTP.

\section{A. Component CoordinatorC}

The component CoordinatorC implements, for the coordinator mote, the MAC layer functions that will interact with upper and lower layers. This component ensures all tasks supported by the coordinator including the synchronization, the data sending, the reception of data and/or resource requests and all the treatment concerning the PMCMTP process. According to the Figure 5 illustrating the graph of the coordinator component, this component is implemented as a set of component modules written in nesC language. These components are connected to each other using adequate interfaces.

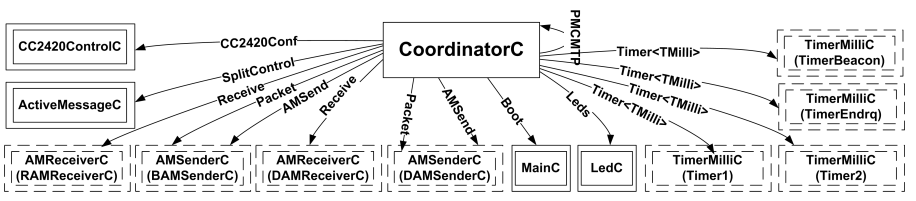

Fig. 5. Coordinator - Component Graph 


\section{B. Component DeviceC}

The component DeviceC implements, for the device motes, the MAC layer functions that will interact with upper and lower layers. This component ensures all tasks supported by a device including the synchronization with the coordinator, the data and/or resource requests sending and the reception of data. According to the Figure 6 illustrating the graph of the Device component, this component is wired to other components of TinyOS library via the adequate interfaces.

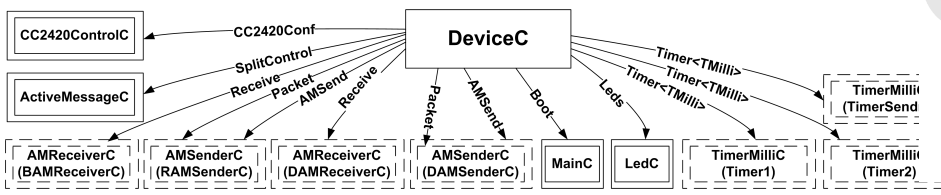

Fig. 6. Device - Component Graph

\section{Interface PMCMTP}

The interface PMCMTP defines required commands and hardware events to ensure the best execution of the RSA algorithm process. Given that the CoordinatorC uses and provides the interface PMCMTP it implements both hardware events and commands.

\section{VALIDATION OF PMCMTP OPERATION}

A WSN testbed consists of a set of sensor nodes deployed in a controlled environment. It is designed to support experimental research in a real-world setting. It provides to researchers a way to test their protocols, algorithms, network issues and applications. For the testbed, a Sensor Network Analyzer SNA, consisting of a Jennic [20] mote, as hardware, and a laptop installing the SNA software is used. The SNA can be configured to scan a set of channel of frequencies by selecting the channels on which we want to perform the scan and its time duration. The functionality of channels scan, shows details of all devices detected using at least one of the selected channels over a specified period of time. For more details about devices and activity on a specific channel, we can select the channel on which we want to capture traffic. Two scenarios are driven to test the PMCMTP operation.

1) Test of the multi-channel access feasibility for a PAN composed by 5 motes: The scenario used to test the feasibility of PMCMTP operation is the following:

- The network is composed of one PAN coordinator and four devices as shown in Figure 7.

- The channel $N^{\circ} 26$ is allocated for control traffic (e.g., network synchronization and resource requests sending and reception).

- The channels $N^{\circ} 11$ and $N^{\circ} 15$ are allocated for data communication traffic.

- During the Request Transmission phase:

- For the first time slot, Device (0x0001) sends, over channel $N^{\circ} 26$, to the coordinator a resource request to communicate with Device (0x0002),
- For the third time slot, Device (0x0003) sends, over channel $N^{\circ} 26$, to the coordinator a resource request to communicate with Device (0x0004).

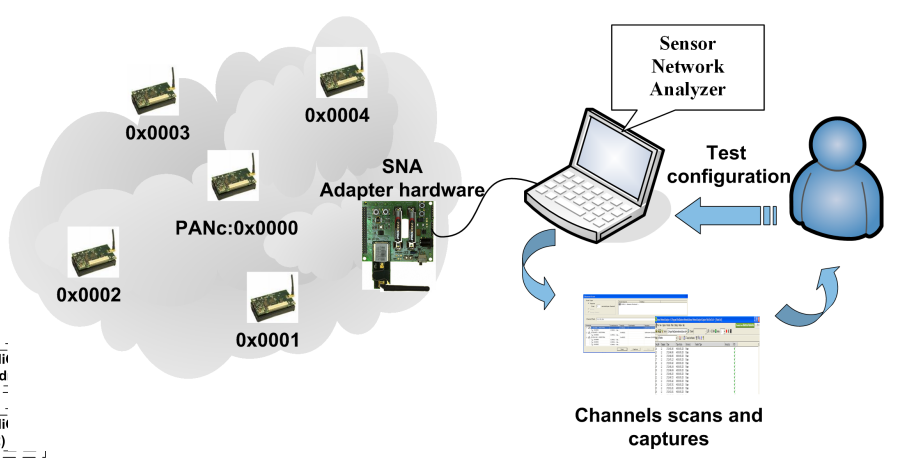

Fig. 7. Network organization for the first scenario

\section{Experimental results}

- Channels scan: We have launched a channel scan operation with a list of three channels $\left(N^{\circ} 11, N^{\circ} 15\right.$ and $\left.N^{\circ} 26\right)$ and for a duration of $1 \mathrm{sec}$ per channel. According to Table I and Figure 8, we note that effectively:

TABLE I

SUMMERY OF THE RESULT OF CHANNELS SCAN

\begin{tabular}{|c|c|c|}
\hline Scanned Channels & PANid & id of source device \\
\hline \hline \multirow{2}{*}{26} & 0x0022 & $\begin{array}{l}\text { 0x0000 } \\
\text { 0x0001 } \\
\text { 0x0003 }\end{array}$ \\
\hline 11 & 0x0022 & 0x0001 \\
\hline 15 & 0x0022 & 0x0003 \\
\hline
\end{tabular}

The channel $N^{\circ} 26$ is used by the coordinator to send beacon frames and by devices (0x0001 and 0x0003) to send their resource requests (See Figure 8(a)). The channel $N^{\circ} 11$ is used by device $(0 x 0001)$ to send data frames (Figure 8(b)). The channel $N^{\circ} 15$ is used by device (0x0003) to send data frames (Figure 8(c));

- Packet timeline: By analyzing the scan of the control channel (i.e., channel $\mathrm{N}^{\circ} 26$ ) over time, we can verify the control part of the PMCMTP process. According to Figure 9, we note that, using the channel $N^{\circ} 26$, periodically:

- the PAN coordinator sends the first beacon to synchronize its network,

- the source nodes according to their $i d$, if they want, they can send a resource request to their coordinator to reserve resource (temporal and spectral resource according to demanded and available resource),

- just before the end of the resource transmission phase, the PAN coordinator launches the RSA process to allocate resource in response to collected resource requests,

- at the end of the resource transmission phase, the PAN coordinator sends the second beacon including the necessary information of served resource requests. 


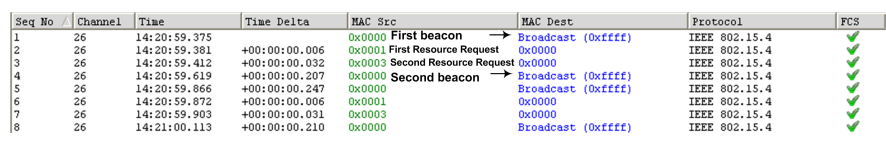

(a) Scan of Control channel $N^{\circ} 26$

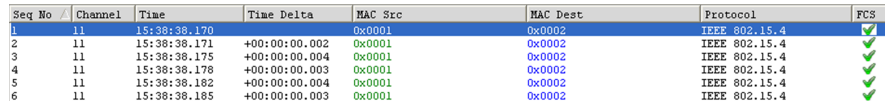

(b) Scan of Data channel $N^{\circ} 11$

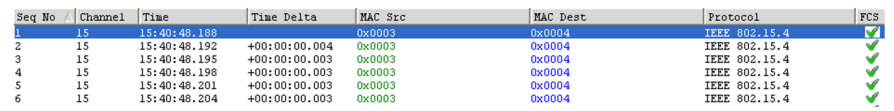

(c) Scan of Data channel $N^{\circ} 15$

Fig. 8. Scan of channels $N^{\circ} 26,11$ and 15

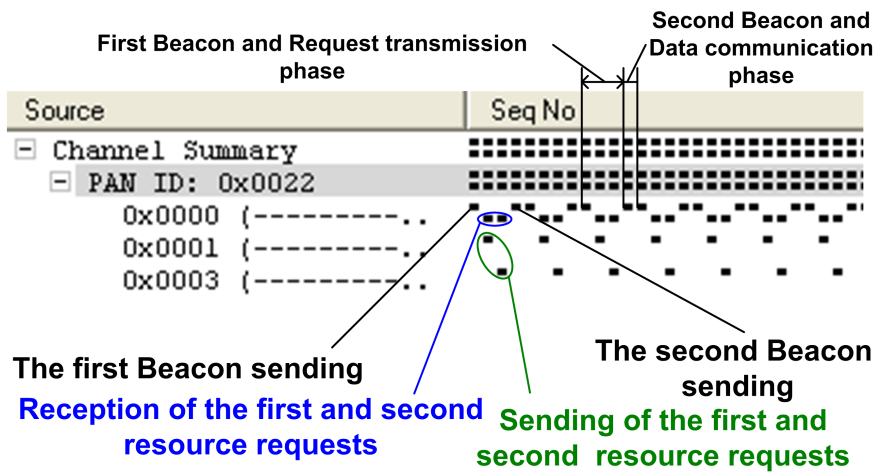

Fig. 9. Packet timeline over channel $N^{\circ} 26$

Compared to Figure 4, illustrating the principle of the PMCMTP operation, these results confirm the correct operation of the synchronization, the resource transmission phase and the data communication phase of the PMCMTP inside a PAN.

2) Test of the multi-channel access feasibility for two PANs of 5 motes each: To generalize the test of PMCMTP operation validity for more than one PAN, we have used the following scenario:

- The network is composed of two PANs, and each is composed of one coordinator and four devices.

- The channels $N^{\circ} 23$ and $N^{\circ} 26$ are respectively allocated to PAN1 (id=0x11) and PAN2 (id=0x22) to ensure control traffic.

- The set of channels $N^{\circ} 11$ and $N^{\circ} 17$ and the set of channels $N^{\circ} 14$ and 20 are respectively allocated to PAN1 and PAN2 to ensure data communication traffic.

- During the Request Transmission phase:

* For the first time slot, Device (0x0001) of PAN1 (resp. of PAN2) sends, over channel $N^{\circ} 23$ (resp. $N^{\circ} 26$ ), to its PAN coordinator a resource request to communicate with Device (0x0002) of PAN1 (resp. of PAN2),

* For the third time slot, Device (0x0003) of PAN1 (resp. of PAN2) sends, over channel $N^{\circ} 23$ (resp. $N^{\circ} 26$ ), to its PAN coordinator a resource request to communicate with Device (0x0004) of PAN1 (resp. of PAN2).

\section{Experimental results}

- Channel scan: We have launched a channel scan operation over six channels $\left(N^{\circ} 11, N^{\circ} 14, N^{\circ} 17, N^{\circ} 20, N^{\circ} 23\right.$ and $N^{\circ} 26$ ) and for a duration of $1 \mathrm{sec}$ per channel.

According to the Table II, Figure 10, Figure 11 and Figure 12, we note that effectively:

TABLE II

SUMMERY OF THE RESULT OF CHANNELS SCAN

\begin{tabular}{|c|c|c|}
\hline Scanned Channels & PANid & id of source device \\
\hline \hline 23 & 0x0011 & $\begin{array}{l}\text { 0x0000 } \\
\text { 0x0001 } \\
\text { 0x0003 }\end{array}$ \\
\hline 11 & 0x0011 & 0x0001 \\
\hline 17 & 0x0011 & 0x0003 \\
\hline \hline 26 & 0x0022 & $\begin{array}{l}\text { 0x0000 } \\
\text { 0x0001 } \\
\text { 0x0003 }\end{array}$ \\
\hline 14 & 0x0022 & 0x0001 \\
\hline 20 & 0x0022 & 0x0003 \\
\hline
\end{tabular}

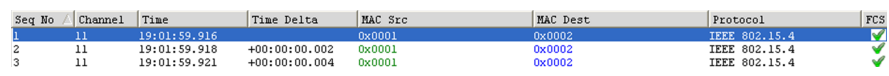

(a) Scan of data channel $N^{\circ} 11$

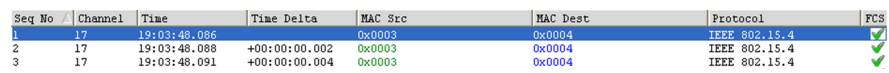

(b) Scan of data channel $N^{\circ} 17$

Fig. 10. Scan of data channels $N^{\circ} 11$ and 17

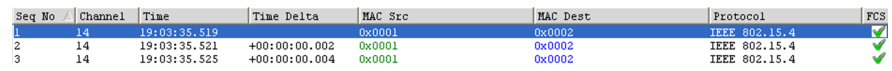

(a) Scan of data channel $N^{\circ} 14$

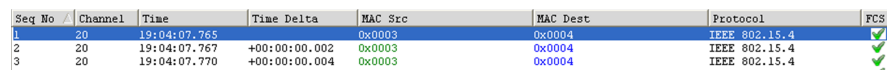

(b) Scan of data channel $N^{\circ} 20$

Fig. 11. Scan of data channels $N^{\circ} 14$ and 20

For PAN1 with PANid equals to 0x0011:

The channel $N^{\circ} 23$ is used by the coordinator (0x0000) to send beacon frames and by devices (0x0001 and 0x0003) to send their resource requests. The channel $N^{\circ} 11$ is used by device $(0 x 0001)$ to send data frames. The channel $N^{\circ} 17$ is used by device (0x0003) to send data frames; For PAN with PANid equals to Ox0022:

The channel $N^{\circ} 26$ is used by the coordinator (0x0000) to send beacon frames and by devices (0x0001 and 0x0003) to send their resource requests. The channel $N^{\circ} 14$ is used by device (0x0001) to send data frames. The channel $N^{\circ} 20$ is used by device (0x0003) to send data frames;

- Packet timeline: As done previously, we have analyzed the scan of the control channels of each PAN (i.e., channel $\mathrm{N}^{\circ} 23$ for PAN1 and channel $\mathrm{N}^{\circ} 26$ for PAN2) 
over time to verify the operation of the control part of the PMCMTP. According to Figure 12, we note that using the channel $\mathrm{N}^{\circ} 23$ (resp. the channel $\mathrm{N}^{\circ} 26$ ): periodically, the coordinator of PAN1 (resp. PAN2) sends the first beacon to synchronize its network, then source nodes according to their $i d$, if they want, they send a resource request to their coordinator to reserve resource. At the end of the resource transmission phase, the coordinator of each PAN sends the second beacon including the necessary information of served resource requests.

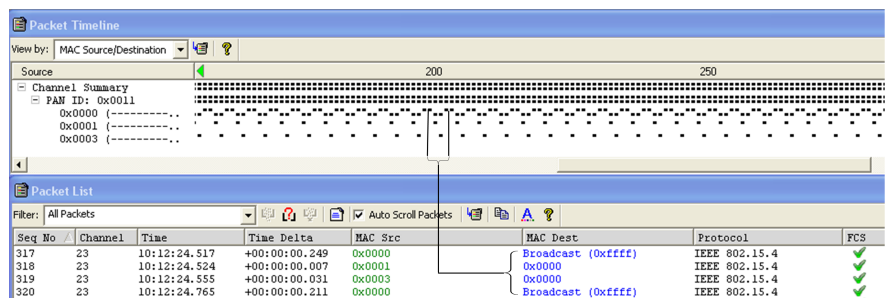

(a) Scan of control channel $N^{\circ} 23$

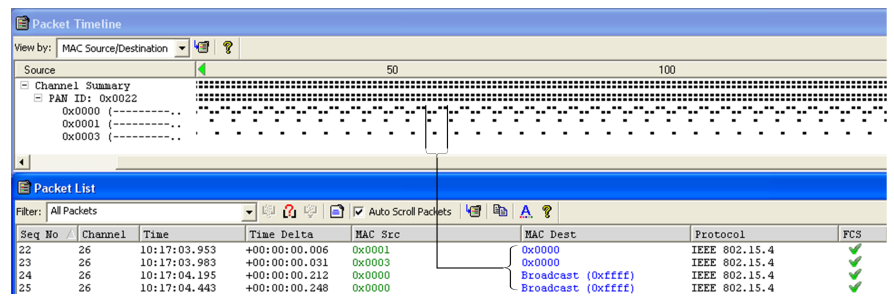

(b) Scan of control channel $N^{\circ} 26$

Fig. 12. Scan of control channels $N^{\circ} 23$ and 26

Compared to Figure 4, illustrating the principle of the PMCMTP operation, these results confirm the correct operation of the PMCMTP inside both PAN1 and PAN2.

\section{CONCLUSION}

In this paper, we have presented the implementation details of the PMCMTP multi-channel multi-time slots MAC protocol in nesC/TinyOS environment for the Crossbow MICAz motes. Two scenarios with respectively one PAN of five motes and two PANs with five motes each one, were used to test, validate and demonstrate the effective operation of the proposed MAC protocol. This set of experiments shows the feasibility of the Multi-channel multi-time slots access mechanism, proving that it can be used in real environments. However, the evaluation of the performance of the PMCMTP, via a realistic testbed setting, remains very encouraged that we will tackle in next work.

\section{REFERENCES}

[1] IEEE 802.15.4a Standard Part 15.4: IEEE Standard for Information Technology, Amendment to IEEE Std 802.15.4-2006, 2007.

[2] X. Wang and T. Berger, "Spatial channel reuse in wireless sensor networks", Springer, vol 14, iss 2, March 2008, pp.133-146.

[3] CC2420 datasheet, inst.eecs.berkeley.edu/ cs150/Documents/CC2420.pdf

[4] Y. Kim, H. Shin, H. Cha, "Y-MAC: An Energy-Efficient Multi-channel MAC Protocol for Dense Wireless Sensor Networks", International Conference on Information Processing in Sensor Networks, 2008, pp.53-63.
[5] O.D. Incel, S. Dulman, and P. Jansen, "Multi-channel Support for Dense Wireless Sensor Networking", The $1^{\text {st }}$ European Conference on Smart Sensing and Context, 2006, the Netherlands, pp.25-27.

[6] X. Chen, P. Han, Q. He, S. Tu and Z. Chen,'A multi-channel MAC protocol for wireless sensor networks", The $16^{\text {th }}$ IEEE International Conference on Computer and Information, 2006, pp.224-229.

[7] K.L. Hieu, D. Henriksson, T. Abdelzaher, "A Practical Multi-Channel Media Access Control Protocol for Wireless Sensor Networks", The International Conference on Information Processing in Sensor Networks, 2008, pp.70-81.

[8] L. Zhiwu, W. Wei, "A Dynamic Multi-radio Multi-channel MAC Protocol for Wireless Sensor Networks", The Second International Conference on Communication Software and Networks, 2010, pp.105-109.

[9] J. Ben Slimane, Y.Q .Song, A. Koubaa, "A Prioritized Multi-Channel Multi-Time slot MAC Protocol For Large-Scale Wireless Sensor Networks", The First International Conference on Communications and Networking, 2009, Hammamet Tunisia.

[10] G. Werner-Allen, P. Swieskowski, M. Welsh, "MoteLab: a wireless sensor network testbed", ISPN, 2005.

[11] D. Johnson, T. Stack, R. Fish, D.M. Flickinger, L. Stoller, R. Ricci, J. Lepreau, "Mobile Emulab: a robotic wireless and sensor network testbed", IEEE INFOCOM, 2006.

[12] N. Baccour, M. Ben Jamaa, D. do Rosario, A. Koubaa, H. Youssef, M. Alves and L. B. Becker, "A TestBed for the Evaluation of Link Quality Estimators in Wireless Sensor Networks", The ACS/IEEE Workshop Future Trends on Ad-hoc and Sensor Networks, 2010.

[13] nescc.sourceforge.net/papers/nesc-ref.pdf

[14] http://www.openautomation.net/uploadsproductos/micaz_datasheet.pdf

[15] http://www.tinyos.net

[16] J. Ben Slimane, Y.Q .Song, A. Koubaa, M. Frikha, "A ThreeTiered Architecture for Large-Scale Wireless Hospital Sensor Networks", the International Workshop on Mobilizing Health Information to Support Healthcare-Related Knowledge Work, 2009, pp.20-31. http://hal.inria.fr/inria-00435508/en/

[17] J. Ben Slimane, Y.Q .Song, A. Koubaa, "Control and data channels allocation for Large-Scale UWB-based WSNs",The First International Conference on Communications and Networking, 2009, Tunisia.

[18] P. Pedreiras, P. Gai, L. Almeida, G.C. Buttazzo, "FTT-Ethernet: A Flexible Real-Time Communication Protocol That Supports Dynamic QoS Management on Ethernet-Based Systems", IEEE Transactions on Industrial Informatics, 2005, Vol.1, Iss.3, pp.162-172.

[19] http://www.astiautomation.ro/produse/Memsic/mib510_en.html

[20] http://www.jennic.com/ 